\title{
PRICES IN THE UTILITY FUNCTION AND DEMAND MONOTONICITY
}

\author{
Emilio Barucci and Filippo Gazzola
}

\begin{abstract}
We analyze utility functions when they depend both on the quantity of the goods consumed by the agent and on the prices of the goods. This approach allows us to model price effects on agents' preferences (e.g. the so-called Veblen effect and the Patinkin formulation). We provide sufficient conditions to observe demand monotonicity and substitution among goods. Power utility functions are investigated: we provide examples of price dependent utility functions that cannot be written as an increasing transformation of a classical utility function dependent only upon quantities.
\end{abstract}

\section{Introduction}

Our main purpose is to discuss in a mathematical framework classical models of consumer theory. In recent years, an increasing interest has been developed for mathematical tools applied to classical theories of utility, market and consumer behavior. In the finance literature, [22, 23] set up refined theories of volatility while in [24] one finds examples of mean-field approaches to modeling in economics and finance. In [4], the authors consider a standard complete market economy with two assets traded in two markets and consider partial differential equations arising in the evaluation of exotic options; they show that the solution of the no-arbitrage partial differential equation is sufficiently regular and that numerical methods can be used for its approximation. Also in the analysis of consumer theory, mathematical methods have provided useful results. In [40] is studied the impact of aesthetic aspects of products in consumer satisfaction: a new nonadditive multiattribute evaluation function is proposed and an application to Kansei evaluation for hand-painted Kutani cups, one of the traditional craft items in Japan, is conducted to illustrate the advantages of the model. In [28], the author considers the problem of ranking linear budget sets with the possibility of different available goods: several axioms and ranking

2010 Mathematics Subject Classification. 35F05, 91B16, $91 \mathrm{~B} 42$.

Key words and phrases. Utility, demand, Veblen effect, transport equation. Received March 12, 2014. 
rules are discussed. The paper [6] proposes a method based on consumer utility for modeling the price thresholds phenomenon that allows for threshold asymmetry, incorporates consumer heterogeneity and uses weekly aggregated brandlevel data; moreover, a method for estimating price elasticity of demand is also included.

The classical consumer theory assumes that the utility only depends on the quantity of the goods consumed by the agent, see e.g. [27]. This hypothesis, that has been motivated during the XX century through an axiomatic microfoundation, allows to fully develop demand theory when agents' preferences are not affected by money or prices. As a consequence, in a general equilibrium framework, prices coordinate agents' decisions signaling the scarcity of the goods, but they do not enter agents' preferences.

This assumption is widely accepted in the literature but there is no complete agreement among researchers. Several arguments have been put forward to insert prices in the utility function. First of all, classical Keynesian macroeconomic theory points out the limits of standard behavioral assumptions to derive money demand, see [31, 32]. Moreover, it is known that price independent utility functions are not a suitable framework to analyze agents' demand for luxury or conspicuous goods, a classical reference being Veblen, see [38]: individuals consume highly conspicuous goods to display their wealth achieving a greater social status, they crave status and the status is enhanced by material display of wealth ("Veblen effect"), for models providing a microfoundation of this behavior see $[2,21,16]$. In these papers, the Veblen effect is the outcome of a signaling model among agents with different status/wealth, in equilibrium the ultimate effect is that wealth and prices enter the utility function. Moreover, as discussed in [30], there are empirical studies on the market demand that reject the main implications derived by a price independent utility (symmetry and negative semidefinitess of the Slutzky matrix), see [39] for applications to collective household models. Finally, as shown in $[34,20]$, there is a line of research on marketing with prices affecting consumer preferences (reference price effect) with applications also in the operational research direction, see $[6,13]$. Following these insights, several authors have suggested to consider utility functions that depend both on prices and on the quantities of goods consumed by the agent, e.g. see $[25,17,1]$. The literature on this topic has investigated two different perspectives.

The first line of research looks for a characterization of price dependent utility functions satisfying certain properties. In this spirit, [17, 19] analyze the demand function when the utility depends upon prices and characterize price dependent utility functions with some given features. $[3,33,11,12,8,7,14,15]$ characterize price dependent utility functions with no money illusion in a neoclassical monetary model $\grave{a}$ la Patinkin (homogeneity of degree zero of the demand in money and prices).

$[5,35,30]$ instead derive empirically verifiable hypotheses on the demand assuming a price dependent utility. The goal is slightly different: authors derive comparative statics results that are alternative to those of the standard optimal 
consumption problem. The new set of comparative statics results may provide a route to address the empirical rejection of the classical model. For an application of price dependent utility to demand of luxury goods see $[29,10,26]$ and $[37,18]$ for an analysis of the saving-consumption problem.

Our analysis belongs to the first strand of literature. Assuming price dependent utility functions, we investigate the agent's demand addressing three main issues: conditions ensuring monotonicity of the demand of a good with respect to its price (no money illusion property), conditions ensuring substitution or complementarity among goods and zero price homogeneity of the demand. We characterize utility functions satisfying these properties. As far as the no money illusion property is concerned, we provide a counterexample to the characterization of utility functions established in the previous literature. We fully analyze power utility functions satisfying the above properties. Our analysis applies to a two goods setting as well as to a multiple goods environment. It turns out that the problem is very rich from a mathematical point of view. Starting with elementary calculus we end up with a linear partial differential inequality which, thanks to a suitable change of unknown function, may be reduced to a simple transport inquelity. It then appears quite natural to try to tackle possible nonlinear problems with the same tools. This is a further purpose of the present paper: to draw the attention of nonlinear analysts on these models. A challenging problem seems to derive a reliable (nonlinear) model equation to describe the Veblen effect.

The paper is organized as follows. In Section 2 we introduce utility functions that also depend on prices. In Section 3 we analyze the two goods setting deriving sufficient conditions to have monotonicity of the demand of a good (the quantity demanded of a good decreases as its price goes up) and for good substitution (the quantity demanded of a good increases as the price of another good increases). In Section 4 we investigate the geometric interpretation of these two demand effects. In Section 5 we characterize two goods utility functions such that the demand functions satisfy the above conditions. In Section 6 we discuss the no money illusion property (demand homogeneity) and we provide a counterexample to the existing results. In Section 7 we analyze power utility functions requiring the above conditions and imposing the homogeneity property. In Section 8 we extend the analysis to more than two goods.

\section{Consumer choices with a price dependent utility function}

We consider the standard optimal consumption problem when the utility function depends on the quantities of the goods consumed by the agent and also on their prices.

The consumer maximizes the utility function $u$ subject to the budget constraint. The utility function depends on the quantity consumed of the $N$ goods $(x)$ and on their prices $(p): u=u(x ; p)$ where $x \in \mathbf{R}_{+}^{N}$ and $p \in \mathbf{R}_{+}^{N}$. Here and in the sequel, $\mathbf{R}_{+}^{N}$ denotes the open sector of $\mathbf{R}^{N}$ of vectors with positive components so that $\mathbf{R}_{+}^{2 N}$ represents the domain of $u$. We assume that the 
agent acts as a price taker so that $p \in \mathbf{R}_{+}^{N}$ are taken as given and he only chooses the quantities $x \in \mathbf{R}_{+}^{N}$.

Given the endowment of the goods $e \in \mathbf{R}_{+}^{N}$, the consumer addresses the following maximization problem

$$
\max _{x \in B} u(x ; p)
$$

where $B$ denotes the consumption bundles that satisfy the budget constraint given the price vector observed in the market:

$$
B=\left\{x \in \mathbf{R}_{+}^{N} ;(x, p)=(e, p)\right\}
$$

where $(\cdot, \cdot)$ denotes the scalar product in $\mathbf{R}^{N}$. We follow classical consumer theory assuming that $u \in C^{2}\left(\mathbf{R}_{+}^{2 N}\right)$ is increasing and strictly concave in each good (diminishing marginal utility):

$$
u_{x_{i}}>0, \quad u_{x_{i} x_{i}}<0 \text { for all } i=1, \ldots, N .
$$

As a consequence, there exists a unique solution $x^{*} \in B$ of the optimization problem (2.1). The solution can be derived via the Lagrange multiplier method: the interior solution $x^{*} \in B$ satisfies

$$
u_{x_{i}}\left(x^{*} ; p\right)=\lambda p_{i} \quad \text { for all } i=1, \ldots, N .
$$

for some Lagrange multiplier $\lambda>0$. Note that the diminishing marginal utility hypothesis is stronger than it is needed to obtain a solution of the optimization problem, the assumption is required in our analysis of power utility, see Section 7 , but can be relaxed in the rest of the paper.

Given the market prices $p \in \mathbf{R}_{+}^{N}$, the (excess) demand function is $z(p)=$ $x^{*}(p)-e$. The demand function obtained when the utility function also depends on prices differs from the classical one. Two main points have been noticed in [17]. If the utility only depends on the quantity of the goods, then the demand function is homogeneous of degree zero in prices, i.e., the solution of the optimization problem (2.1) does not change if the price vector is multiplied by a positive constant. This is not necessarily the case if the utility function also depends on prices: as a matter of fact, prices affect the utility and a homothety of the price vector and wealth does not change the budget constraint but it may affect preferences and the utility function.

When the utility function only depends on the quantity of the goods consumed by the agent, comparative static results can be developed through the classical Slutzky equation which is made up of two components: the income and the substitution effect. When the utility function also depends on prices, [17] has shown that the effect of the change of the price of a good on the quantity demanded of another good is provided by three components: the income and substitution effects and the price effect that reflects the direct effect of the price change on preferences and on the demand. 


\section{The two goods case}

The unique interior solution $x^{*} \in B$ to the optimization problem (2.1) may be found via the Lagrange multiplier method. When $N=2, x^{*} \in B$ satisfies

$$
u_{x_{1}}\left(x^{*} ; p\right)=\lambda p_{1} \quad u_{x_{2}}\left(x^{*} ; p\right)=\lambda p_{2}
$$

for some Lagrange multiplier $\lambda>0$. The two conditions yield

$$
p_{2} u_{x_{1}}\left(x^{*} ; p\right)-p_{1} u_{x_{2}}\left(x^{*} ; p\right)=0 .
$$

The optimal consumption $x^{*}$ is a function of the price vector and of the wealth $\omega=(p, e): x^{*}=x^{*}(p, \omega)$. [17] has shown that a generalized Slutsky equation holds:

$$
\frac{\partial x_{i}^{*}}{\partial p_{j}}=S_{i j}-x_{j}^{*} \frac{\partial x_{i}^{*}}{\partial \omega}+\frac{u_{p_{j}}}{\lambda} \frac{\partial x_{i}^{*}}{\partial \omega} .
$$

The effect of a change of the price of good $j$ on the quantity demanded of good $i$ is made up of three components: $S_{i j}$ (the effect of a change of the price $j$ on the quantity demanded of good $i$ along the indifference curve) is the substitution effect, the second is the income effect, the third component is denominated the price offsetting income component. Only the first two components are detected when the utility function merely depends on the quantities of the goods.

Without loss of generality, in what follows we assume that the endowment is fixed in terms of wealth $(\omega>0)$ and we characterize utility functions $u$ that imply one of the two following behaviors as $p_{1}$ varies:

$$
\text { (C) } \frac{\partial x_{1}^{*}}{\partial p_{1}} \leq 0, \quad \text { (S) } \frac{\partial x_{2}^{*}}{\partial p_{1}} \geq 0 .
$$

Symmetric conditions are required as $p_{2}$ varies. If we differentiate totally with respect to $p_{1}$ the budget constraint $\left(x^{*}(p), p\right)=\omega$ we obtain

$$
x_{1}^{*}+p_{1} \frac{\partial x_{1}^{*}}{\partial p_{1}}+p_{2} \frac{\partial x_{2}^{*}}{\partial p_{1}}=0 \text { for all } p \in \mathbf{R}_{+}^{2} .
$$

This readily shows that

$$
(\mathrm{S}) \Rightarrow(\mathrm{C})
$$

In case $(\mathrm{C})$ we observe that the quantity demanded of a good decreases as its price goes up. We do not make any assumption on the demand of the other good. Also in case (S) the quantity demanded of a good decreases with respect to its price (due to (3.5)) but now there is substitution between goods: as the price of the first good increases, the quantity demanded of the first good decreases and the quantity demanded of the second good increases, i.e., the sign of (3.2) is positive. In case (C) instead we allow for complementarity between the two goods, i.e., the quantity demanded of both goods may decrease as the price of the first good increases and therefore we refer to this demand behavior as the 
complementarity case. Note that we address complementarity and substitution between goods considering the total effect on the demand of a good associated with the change of the price of the good or the price of another good ${ }^{1}$.

We now want to characterize utility functions in case $(C)$ and $(\mathrm{S})$. Let us start with case $(\mathbf{S})$. Omitting wealth, which is fixed, we have that $x^{*}=x^{*}(p)$. Let us differentiate (3.1) totally with respect to $p_{1}$, we obtain

$$
\begin{aligned}
& p_{2} u_{x_{1} x_{1}}\left(x^{*}(p) ; p\right) \frac{\partial x_{1}^{*}}{\partial p_{1}}+p_{2} u_{x_{1} x_{2}}\left(x^{*}(p) ; p\right) \frac{\partial x_{2}^{*}}{\partial p_{1}} \\
& \quad+p_{2} u_{x_{1} p_{1}}\left(x^{*}(p) ; p\right)-u_{x_{2}}\left(x^{*}(p) ; p\right)+ \\
& \quad-p_{1} u_{x_{1} x_{2}}\left(x^{*}(p) ; p\right) \frac{\partial x_{1}^{*}}{\partial p_{1}}-p_{1} u_{x_{2} x_{2}}\left(x^{*}(p) ; p\right) \frac{\partial x_{2}^{*}}{\partial p_{1}} \\
& \quad-p_{1} u_{x_{2} p_{1}}\left(x^{*}(p) ; p\right)=0 \text { for all } p \in \mathbf{R}_{+}^{2} .
\end{aligned}
$$

By combining (3.4) with (3.6), at the point $\left(x^{*}(p) ; p\right)$ we have

$$
\begin{aligned}
& p_{2} u_{x_{1} p_{1}}-p_{1} u_{x_{2} p_{1}}-u_{x_{2}}+x_{1}^{*} u_{x_{1} x_{2}}-\frac{p_{2} x_{1}^{*}}{p_{1}} u_{x_{1} x_{1}} \\
& =\frac{1}{p_{1}}\left(p_{2}^{2} u_{x_{1} x_{1}}-2 p_{1} p_{2} u_{x_{1} x_{2}}+p_{1}^{2} u_{x_{2} x_{2}}\right) \frac{\partial x_{2}^{*}}{\partial p_{1}} \quad \text { for all } p \in \mathbf{R}_{+}^{2} .
\end{aligned}
$$

In the $\left(x_{1}, x_{2}\right)$-plane the budget constraint $(x, p)=\omega$ is a segment containing the vector $Q=\left(-p_{2}, p_{1}\right)$. Using this vector we may rewrite $(3.7)$ as

$$
p_{2} u_{x_{1} p_{1}}-p_{1} u_{x_{2} p_{1}}-u_{x_{2}}+x_{1}^{*} u_{x_{1} x_{2}}-\frac{p_{2} x_{1}^{*}}{p_{1}} u_{x_{1} x_{1}}=\frac{1}{p_{1}} \frac{\partial^{2} u}{\partial Q^{2}} \frac{\partial x_{2}^{*}}{\partial p_{1}} \quad \text { for all } p \in \mathbf{R}_{+}^{2} \text {. }
$$

Note that $\frac{\partial^{2} u}{\partial Q^{2}}$ represents the second derivative of $u$ in the direction of the constraint evaluated at the maximum point $x^{*}$ of $u$ subject to the constraint, it coincides with bordered Hessian matrix of the utility maximization problem evaluated at $x^{*}$ and therefore is non positive. Hence, in view of assumption (S), at the point $\left(x^{*}(p) ; p\right)$ we find

$$
-\frac{p_{2} x_{1}^{*}}{p_{1}} u_{x_{1} x_{1}}+p_{2} u_{x_{1} p_{1}}-u_{x_{2}}+x_{1}^{*} u_{x_{1} x_{2}}-p_{1} u_{x_{2} p_{1}} \leq 0 \text { for all } p \in \mathbf{R}_{+}^{2}
$$

Not only it appears difficult to provide necessary and sufficient conditions for (3.8) to hold but also it is not straightforward how to check (3.8) even in simple cases. We are so led to find sufficient conditions which imply (3.8), the

${ }^{1}[17,19]$ instead refer to demand changes along the indifference curve (Slutsky effect) providing comparative statics results and conditions ensuring symmetry and negative semidefiniteness of the Slutsky matrix. 
simplest one being the one which requires (3.8) to be satisfied at any point $(x ; p) \in \mathbf{R}_{+}^{4}$ and not just at $\left(x^{*}(p) ; p\right)$ for all $p \in \mathbf{R}_{+}^{2}$. Therefore, we get

$$
-\frac{p_{2} x_{1}}{p_{1}} u_{x_{1} x_{1}}+p_{2} u_{x_{1} p_{1}}-u_{x_{2}}+x_{1} u_{x_{1} x_{2}}-p_{1} u_{x_{2} p_{1}} \leq 0 \text { for all }(x ; p) \in \mathbf{R}_{+}^{4} .
$$

The differential inequality (3.9) gives a sufficient condition for the substitution effect $(\mathrm{S})$ to hold and it will be called strong substitution (SS). As it will become clear in Section 7, this stronger condition maintains some form of linearity and makes it simpler to verify the condition.

In case (C), by combining (3.4) and (3.6), at the point $\left(x^{*}(p) ; p\right)$ we have

$$
\begin{aligned}
& p_{2} u_{x_{1} p_{1}}-p_{1} u_{x_{2} p_{1}}-u_{x_{2}}-x_{1}^{*} u_{x_{1} x_{2}}+\frac{p_{1} x_{1}^{*}}{p_{2}} u_{x_{2} x_{2}} \\
& =-\frac{1}{p_{2}}\left(p_{2}^{2} u_{x_{1} x_{1}}-2 p_{1} p_{2} u_{x_{1} x_{2}}+p_{1}^{2} u_{x_{2} x_{2}}\right) \frac{\partial x_{1}^{*}}{\partial p_{1}} \quad \text { for all } p \in \mathbf{R}_{+}^{2} .
\end{aligned}
$$

As for (3.7), we may rewrite (3.10) as

$$
p_{2} u_{x_{1} p_{1}}-p_{1} u_{x_{2} p_{1}}-u_{x_{2}}-x_{1}^{*} u_{x_{1} x_{2}}+\frac{p_{1} x_{1}^{*}}{p_{2}} u_{x_{2} x_{2}}=-\frac{1}{p_{2}} \frac{\partial^{2} u}{\partial Q^{2}} \frac{\partial x_{1}^{*}}{\partial p_{1}} \quad \text { for all } p \in \mathbf{R}_{+}^{2} \text {. }
$$

By combining this with the assumption $(\mathrm{C})$, at the point $\left(x^{*}(p) ; p\right)$ we find

$$
-x_{1}^{*} u_{x_{1} x_{2}}+p_{2} u_{x_{1} p_{1}}-u_{x_{2}}+\frac{p_{1} x_{1}^{*}}{p_{2}} u_{x_{2} x_{2}}-p_{1} u_{x_{2} p_{1}} \leq 0 \text { for all } p \in \mathbf{R}_{+}^{2} \text {. }
$$

Again, a sufficient condition may be obtained by requiring (3.11) to be satisfied at any point $(x ; p) \in \mathbf{R}_{+}^{4}$ and not just at $\left(x^{*}(p) ; p\right)$ for all $p \in \mathbf{R}_{+}^{2}$. Such a condition reads

$$
\begin{array}{r}
-x_{1} u_{x_{1} x_{2}}(x ; p)+p_{2} u_{x_{1} p_{1}}(x ; p)-u_{x_{2}}(x ; p) \\
+\frac{p_{1} x_{1}}{p_{2}} u_{x_{2} x_{2}}(x ; p)-p_{1} u_{x_{2} p_{1}}(x ; p) \leq 0
\end{array}
$$

for all $(x ; p) \in \mathbf{R}_{+}^{4}$. This condition will be called strong complementarity (SC).

\section{A geometric interpretation}

In this section we obtain the results of the previous one from a geometric point of view. The reason for this analysis is that a geometric interpretation of the conditions (SS) and (SC) will be useful in Section 8 to extend our results to the case of many goods $(N \geq 2)$.

Fixed $p \in \mathbf{R}_{+}^{2}$, consider the level line of the utility function for the optimum $x^{*}$ :

$$
\ell=\left\{x \in \mathbf{R}_{+}^{2} ; u(x ; p)=u\left(x^{*} ; p\right)\right\} .
$$

For all $x \in \ell$ we may determine the sign of $p_{2} u_{x_{1}}(x ; p)-p_{1} u_{x_{2}}(x ; p)$. We prove the following result. 
LEMMA 4.1. Let $x \in \ell$ :

(i) if $x_{2}>x_{2}^{*}$ then $p_{2} u_{x_{1}}(x ; p)-p_{1} u_{x_{2}}(x ; p)>0$,

(ii) if $x_{2}<x_{2}^{*}$ then $p_{2} u_{x_{1}}(x ; p)-p_{1} u_{x_{2}}(x ; p)<0$.

Proof. For a fixed $p \in \mathbf{R}_{+}^{2}$, the utility function can be interpreted as a function depending only on $x \in \mathbf{R}_{+}^{2}, u=u(x)$. We embed $\mathbf{R}^{2}$ into $\mathbf{R}^{3}$ in such a way that $x_{1}$ is the horizontal axis, $x_{2}$ is the vertical axis and $x_{3}$ is the axis orthogonal to the plane so that $\left(x_{1}, x_{2}, x_{3}\right)$ is a direct triple. In $\mathbf{R}^{3}$ we can compute the vector product $\nabla u(x) \wedge p$. The triple of vectors $(\nabla u(x), p, \nabla u(x) \wedge p)$ also forms a direct triple. Its component along the $x_{3}$-axis is precisely $p_{2} u_{x_{1}}(x)-$ $p_{1} u_{x_{2}}(x)$.

Assume first that $x_{2}>x_{2}^{*}$ and consider the straight line $R$ containing $x \in \ell$ and being parallel to $B$. The vector $p$ is orthogonal to $R$. The level line $\ell$ crosses $R$ at $x$. Moreover, $\ell$ is above $R$ in the strip $\left\{y \in \mathbf{R}_{+}^{2} ; y_{1}<x_{1}\right\}$ whereas $R$ is above $\ell$ in the region $\left\{y \in \mathbf{R}_{+}^{2} ; y_{1}>x_{1}\right\}$. Therefore, since $\nabla u(x)$ is orthogonal to $\ell$ at $x$, the vector $p$ is above the vector $\nabla u(x)$. This shows that $\nabla u(x) \wedge p$ has a positive $x_{3}$-component. The case $x_{2}<x_{2}^{*}$ is similar, one just has to change all the signs.

Let us evaluate the demand function as $p_{1}$ and the relative price $\frac{p_{1}}{p_{2}}$ change. If the price $p_{1}$ increases to $p_{1}+\varepsilon$ for some $\varepsilon>0$, then the new budget constraint reads

$$
B_{\varepsilon}=\left\{x \in \mathbf{R}_{+}^{2} ;\left(p_{1}+\varepsilon\right) x_{1}+p_{2} x_{2}=\omega\right\} .
$$

Let $x^{*}=\left(x_{1}^{*}, x_{2}^{*}\right) \in B$ denote the solution to $(2.1)$ and $x^{\varepsilon}=\left(x_{1}^{\varepsilon}, x_{2}^{\varepsilon}\right) \in B_{\varepsilon}$ be the solution to the optimization problem

$$
\max _{x \in B_{\varepsilon}} u\left(x ; p_{1}+\varepsilon, p_{2}\right) .
$$

Complementarity and substitution, as defined in (3.3), read

$$
\text { (C) } x_{1}^{\varepsilon} \leq x_{1}^{*}, \quad(\mathrm{~S}) x_{2}^{\varepsilon} \geq x_{2}^{*} \text {. }
$$

In the more restricted situation $(\mathrm{S})$, where $x_{2}^{\varepsilon} \geq x_{2}^{*}$, consider the point $\bar{x} \in B_{\varepsilon}$ having the same ordinate as $x^{*}$, so that

$$
\bar{x}_{1}=\frac{p_{1}}{p_{1}+\varepsilon} x_{1}^{*}, \quad \bar{x}_{2}=x_{2}^{*} .
$$

Then, from Lemma 4.1 applied to $\bar{x}$ we obtain

$$
p_{2} u_{x_{1}}\left(\bar{x} ; p_{1}+\varepsilon, p_{2}\right)-\left(p_{1}+\varepsilon\right) u_{x_{2}}\left(\bar{x} ; p_{1}+\varepsilon, p_{2}\right) \leq 0 .
$$

Taking into account (4.2), this yields (for all $\varepsilon>0$ )

$$
p_{2} u_{x_{1}}\left(\frac{p_{1}}{p_{1}+\varepsilon} x_{1}^{*}, x_{2}^{*} ; p_{1}+\varepsilon, p_{2}\right)-\left(p_{1}+\varepsilon\right) u_{x_{2}}\left(\frac{p_{1}}{p_{1}+\varepsilon} x_{1}^{*}, x_{2}^{*} ; p_{1}+\varepsilon, p_{2}\right) \leq 0 \text {. }
$$


Inequality (4.3) is equivalent to $(\mathrm{S})$. (SS) requires (4.3) to be satisfied at any point $(x ; p) \in \mathbf{R}_{+}^{4}$ and not just at $\left(x^{*}(p) ; p\right)$ for all $p \in \mathbf{R}_{+}^{2}$. So, for any $(x ; p) \in \mathbf{R}_{+}^{4}$ we consider the function

$$
\varphi(\varepsilon):=p_{2} u_{x_{1}}\left(\frac{p_{1}}{p_{1}+\varepsilon} x_{1}, x_{2} ; p_{1}+\varepsilon, p_{2}\right)-\left(p_{1}+\varepsilon\right) u_{x_{2}}\left(\frac{p_{1}}{p_{1}+\varepsilon} x_{1}, x_{2} ; p_{1}+\varepsilon, p_{2}\right)
$$

defined for all $\varepsilon \geq 0$. Notice that if $x=x^{*}(p)$ we have $\varphi(0)=0$. Therefore, the extension of (4.3) to any point $(x ; p) \in \mathbf{R}_{+}^{4}$ reads $\varphi(\varepsilon) \leq \varphi(0)$ for all $\varepsilon>0$. In turn, this happens if and only if $\varphi^{\prime}(0) \leq 0$, namely if (3.9) holds. We point out that the two conditions $\varphi(\varepsilon) \leq \varphi(0)$ for all $\varepsilon>0$ and $\varphi^{\prime}(0) \leq 0$ are not equivalent as a characterization of $(\mathbf{S})$, they are equivalent in our situation because (3.9) must hold true for any $x \in \mathbf{R}_{+}^{2}$. so that

In case $(\mathrm{C})$ we consider the point $\tilde{x} \in B_{\varepsilon}$ having the same abscissa as $x^{*}$,

$$
\tilde{x}_{1}=x_{1}^{*}, \quad \tilde{x}_{2}=x_{2}^{*}-\frac{\varepsilon}{p_{2}} x_{1}^{*} .
$$

According to Lemma 4.1 applied to $\tilde{x}$, we have that $(\mathrm{C})$ is characterized by

$$
p_{2} u_{x_{1}}\left(\tilde{x} ; p_{1}+\varepsilon, p_{2}\right)-\left(p_{1}+\varepsilon\right) u_{x_{2}}\left(\tilde{x} ; p_{1}+\varepsilon, p_{2}\right) \leq 0 \text { for all } \varepsilon>0 .
$$

In order to obtain (SC) we require that this inequality holds for any $(x ; p) \in \mathbf{R}_{+}^{4}$ and we obtain

$$
\begin{aligned}
\psi(\varepsilon):= & p_{2} u_{x_{1}}\left(x_{1}, x_{2}-\frac{\varepsilon}{p_{2}} x_{1} ; p_{1}+\varepsilon, p_{2}\right) \\
& -\left(p_{1}+\varepsilon\right) u_{x_{2}}\left(x_{1}, x_{2}-\frac{\varepsilon}{p_{2}} x_{1} ; p_{1}+\varepsilon, p_{2}\right) \leq 0
\end{aligned}
$$

for all $\varepsilon>0$. In turn, this occurs (independently of $x_{1}$ and $x_{2}$ ) if and only if $\psi^{\prime}(0) \leq 0$, namely if and only if (3.12) holds.

Conditions ensuring strong substitution or strong complementarity of the demand function strictly depend on the assumption that the utility function depends upon prices. If this is not the case, then (SS) becomes

$$
\varphi(\varepsilon):=p_{2} u_{x_{1}}\left(\frac{p_{1}}{p_{1}+\varepsilon} x_{1}, x_{2}\right)-\left(p_{1}+\varepsilon\right) u_{x_{2}}\left(\frac{p_{1}}{p_{1}+\varepsilon} x_{1}, x_{2}\right) \leq 0 \text { for all } \varepsilon>0 \text {. }
$$

This occurs if and only if $\varphi^{\prime}(0) \leq 0$, namely

$$
-\frac{p_{2} x_{1}}{p_{1}} u_{x_{1} x_{1}}\left(x_{1}, x_{2}\right)-u_{x_{2}}\left(x_{1}, x_{2}\right)+x_{1} u_{x_{1} x_{2}}\left(x_{1}, x_{2}\right) \leq 0 \text { for all }\left(x_{1}, x_{2}\right) \in \mathbf{R}_{+}^{2} \text {. }
$$

In view of (2.3), it is clear that (4.4) does not hold under the extreme condition where $\frac{p_{2}}{p_{1}}$ is very large (either $p_{2}$ very large or $p_{1}$ very small). As a consequence, 
there is no utility function that satisfies (SS) for all vectors $(x ; p) \in \mathbf{R}_{+}^{4}$. Instead, when the utility function also depends upon prices we may find utility functions that satisfy (SS) in the full space.

We conclude this section by comparing the two differential inequalities which characterize (SC) and (SS). For all $(x ; p) \in \mathbf{R}_{+}^{4}$ we define

$$
\begin{aligned}
\Phi(x, p):= & p_{2} u_{x_{1} p_{1}}(x ; p)-p_{1} u_{x_{2} p_{1}}(x ; p)-u_{x_{2}}(x ; p) \\
& +x_{1} u_{x_{1} x_{2}}(x ; p)-\frac{p_{2} x_{1}}{p_{1}} u_{x_{1} x_{1}}(x ; p)
\end{aligned}
$$

and

$$
\begin{aligned}
\Psi(x, p):= & p_{2} u_{x_{1} p_{1}}(x ; p)-p_{1} u_{x_{2} p_{1}}(x ; p)-u_{x_{2}}(x ; p) \\
& -x_{1} u_{x_{1} x_{2}}(x ; p)+\frac{p_{1} x_{1}}{p_{2}} u_{x_{2} x_{2}}(x ; p) .
\end{aligned}
$$

According to (3.9) and (3.12), we have

$$
\begin{gathered}
\text { strong substitution (SS) } \Leftrightarrow \Phi(x, p) \leq 0 \text { in } \mathbf{R}_{+}^{4}, \\
\text { strong complementarity (SC) } \Leftrightarrow \Psi(x, p) \leq 0 \text { in } \mathbf{R}_{+}^{4} .
\end{gathered}
$$

We only interpret the difference between $\Phi$ and $\Psi$, conditions on $p_{2}$ are similar see (5.2) and (5.4) below. Consider the restriction of $u, \Phi$ and $\Psi$ to the $\left(x_{1}, x_{2}\right)$ plane where the budget constraint $(x, p)=\omega$ is a segment containing the vector $Q=\left(-p_{2}, p_{1}\right)$, it is easy to show that

$$
\Psi(x, p)-\Phi(x, p)=\frac{x_{1}}{p_{1} p_{2}} \frac{\partial^{2} u}{\partial Q^{2}}(x ; p) .
$$

Let us recall that $(\mathrm{S})$ and $(\mathrm{C})$ require to satisfy (3.9) and (3.12) only at the couple $\left(x^{*}(p) ; p\right)$. At the optimum $x^{*}=x^{*}(p)$ which maximizes $u$ over $B$ (recall that $p$ is fixed), the second derivative in the direction of the vector constraint $Q$ is non positive:

$$
\frac{\partial^{2} u}{\partial Q^{2}}\left(x^{*}\right) \leq 0
$$

Our sufficient conditions require (4.8) to hold on $\mathbf{R}^{2}$ and therefore (SS) implies (CC) providing a generalization of (3.5).

\section{Characterization of the utility function}

In this section we characterize utility functions for the two goods setting satisfying conditions (SS) or (SC). The first step consists in rewriting inequality (3.9) as

$$
p_{2}\left(p_{1} u_{p_{1}}-x_{1} u_{x_{1}}+u\right)_{x_{1}}-p_{1}\left(p_{1} u_{p_{1}}-x_{1} u_{x_{1}}+u\right)_{x_{2}} \leq 0
$$


and, in a dual way (if $p_{2}$ increases), we obtain the differential inequality

$$
p_{1}\left(p_{2} u_{p_{2}}-x_{2} u_{x_{2}}+u\right)_{x_{2}}-p_{2}\left(p_{2} u_{p_{2}}-x_{2} u_{x_{2}}+u\right)_{x_{1}} \leq 0 .
$$

Inequalities (5.1)-(5.2) characterize case (SS).

Similarly, we may rewrite inequality (3.12) as

$$
p_{2}\left(p_{2} u_{p_{1}}-x_{1} u_{x_{2}}\right)_{x_{1}}-p_{1}\left(p_{2} u_{p_{1}}-x_{1} u_{x_{2}}\right)_{x_{2}} \leq 0
$$

and, in a dual way (if $p_{2}$ increases), we obtain the differential inequality

$$
p_{1}\left(p_{1} u_{p_{2}}-x_{2} u_{x_{1}}\right)_{x_{2}}-p_{2}\left(p_{1} u_{p_{2}}-x_{2} u_{x_{1}}\right)_{x_{1}} \leq 0 .
$$

Inequalities (5.3)-(5.4) characterize case (SC).

Before stating our results, we point out that we have no boundary conditions (such as Cauchy conditions or Dirichlet conditions) for the utility function $u$ and this leaves many degrees of freedom. We do not even have other kinds of constraints, such as assumptions on the behavior of $u$ in extremal conditions, for instance as $\left(p_{1}, p_{2}\right) \rightarrow(0,0)$; in this case, it could happen that $u$ blows up at infinity. As a consequence, integration in a neighborhood of $\left(p_{1}, p_{2}\right)=(0,0)$ is extremely delicate. This suggests to fix a boundary condition for $p_{1}=1$ and $p_{2}=1$. This is the reason of the appearance of the integral $\int_{1}^{p_{i}}$ in formulas (5.6)-(5.7) and (5.12)-(5.13) below.

In the strong substitution case (SS) the following Theorem is obtained.

Theorem 5.1. Assume that $u \in C^{2}\left(\mathbf{R}_{+}^{4}\right)$ satisfies (5.1). Then there exists a function

$$
h \in C^{1}\left(\mathbf{R}_{+}^{4}\right) \quad \text { such that } h=h(\xi) \quad \text { satisfies } \quad \frac{\partial h}{\partial \xi_{1}} \leq 0
$$

and a function $g \in C^{2}\left(\mathbf{R}_{+}^{3}\right)$ such that $u$ has the form

$$
u(x ; p)=\frac{1}{p_{1}} \int_{1}^{p_{1}} h\left(\frac{p_{1} x_{1}}{t^{2}}, p_{1} x_{1}+p_{2} x_{2}, t, p_{2}\right) d t+\frac{g\left(p_{1} x_{1}, x_{2}, p_{2}\right)}{p_{1}} .
$$

Similarly, if $u \in C^{2}\left(\mathbf{R}_{+}^{4}\right)$ satisfies (5.2), then $u$ has the form

$$
u(x ; p)=\frac{1}{p_{2}} \int_{1}^{p_{2}} \tilde{h}\left(\frac{p_{2} x_{2}}{t^{2}}, p_{1} x_{1}+p_{2} x_{2}, t, p_{1}\right) d t+\frac{\tilde{g}\left(p_{2} x_{2}, x_{1}, p_{1}\right)}{p_{2}},
$$

where $\tilde{h}$ satisfies (5.5) and $\tilde{g} \in C^{2}\left(\mathbf{R}_{+}^{3}\right)$. Therefore, in case of strong substitution $(S S), u$ is necessarily written in both the two forms (5.6) and (5.7).

Proof. Setting $w:=p_{1} u_{p_{1}}-x_{1} u_{x_{1}}+u$, (5.1) reads

$$
p_{2} w_{x_{1}}-p_{1} w_{x_{2}} \leq 0
$$


This is a (first order) transport partial differential inequality. In order to solve (5.8), we make the change of variables

$$
\left\{\begin{array} { l } 
{ \xi _ { 1 } = \frac { x _ { 1 } } { p _ { 2 } } } \\
{ \xi _ { 2 } = p _ { 1 } x _ { 1 } + p _ { 2 } x _ { 2 } } \\
{ \xi _ { 3 } = p _ { 1 } } \\
{ \xi _ { 4 } = p _ { 2 } }
\end{array} \quad \left\{\begin{array}{l}
x_{1}=\xi_{1} \xi_{4} \\
x_{2}=\frac{\xi_{2}}{\xi_{4}}-\xi_{1} \xi_{3} \\
p_{1}=\xi_{3} \\
p_{2}=\xi_{4}
\end{array}\right.\right.
$$

and we put $h\left(\xi_{1}, \xi_{2}, \xi_{3}, \xi_{4}\right)=w\left(\xi_{1} \xi_{4}, \frac{\xi_{2}}{\xi_{4}}-\xi_{1} \xi_{3}, \xi_{3}, \xi_{4}\right)$. Note that the change of variables (5.9) is legitime since it defines a one-to-one map between $\left\{(x ; p) \in \mathbf{R}_{+}^{4}\right\}$ and $A=\left\{\xi \in \mathbf{R}_{+}^{4} ; \xi_{2}>\xi_{1} \xi_{3} \xi_{4}\right\}$ so that the function $h$ is defined in $A$. With this change of variables, (5.8) becomes

$$
h_{\xi_{1}}\left(\xi_{1}, \xi_{2}, \xi_{3}, \xi_{4}\right) \leq 0 .
$$

We have so shown that if $w$ solves (5.8) then there exists a function $h$ satisfying (5.5) such that

$$
w\left(x_{1}, x_{2}, p_{1}, p_{2}\right)=h\left(\frac{x_{1}}{p_{1}}, p_{1} x_{1}+p_{2} x_{2}, p_{1}, p_{2}\right) .
$$

Hence, we are led to solve

$$
p_{1} u_{p_{1}}(x ; p)-x_{1} u_{x_{1}}(x ; p)+u(x ; p)=h\left(\frac{x_{1}}{p_{1}}, p_{1} x_{1}+p_{2} x_{2}, p_{1}, p_{2}\right) .
$$

In order to solve (5.10) we freeze $x_{2}$ and $p_{2}$ and consider them as constant parameters. In the $\left(x_{1}, p_{1}\right)$-plane, the characteristic curves of the linear hyperbolic equation (5.10) are the hyperbolas $x_{1} p_{1}=\gamma>0$. This suggests to perform the change of variables

$$
\left\{\begin{array} { l } 
{ y = p _ { 1 } } \\
{ z = p _ { 1 } x _ { 1 } }
\end{array} \quad \left\{\begin{array}{l}
p_{1}=y \\
x_{1}=\frac{z}{y}
\end{array}\right.\right.
$$

and to put $u\left(x_{1}, p_{1}\right)=f(y, z)=f\left(p_{1}, p_{1} x_{1}\right)$, the variables $x_{2}$ and $p_{2}$ being frozen. Then, (5.10) becomes

$$
(y f(y, z))_{y}=h\left(\frac{z}{y^{2}}, z+p_{2} x_{2}, y, p_{2}\right) .
$$

Integrating (5.11) over $[1, y]$ (for any $y>0$ ) we obtain

$$
f(y, z)=\frac{1}{y} \int_{1}^{y} h\left(\frac{z}{t^{2}}, z+p_{2} x_{2}, t, p_{2}\right) d t+\frac{g\left(z, x_{2}, p_{2}\right)}{y},
$$


where $g\left(z, x_{2}, p_{2}\right)=f(1, z)$ (recall the frozen variables $x_{2}$ and $\left.p_{2}\right)$. In turn, back to the original variables, the latter expression yields (5.6).

In a dual way, if we freeze $x_{1}$ and $p_{1}$, we find that $u$ has the form (5.7).

In the strong complementarity case (SC) the following Theorem is obtained.

THEOREM 5.2. Assume that $u \in C^{2}\left(\mathbf{R}_{+}^{4}\right)$ satisfies (5.3). There exists a function $h \in C^{1}\left(\mathbf{R}_{+}^{4}\right)$ satisfying (5.5) and a function $g \in C^{2}\left(\mathbf{R}_{+}^{3}\right)$ such that $u$ has the form

$$
u(x ; p)=\frac{1}{p_{2}} \int_{1}^{p_{1}} h\left(\frac{x_{1}}{t}, p_{1} x_{1}+p_{2} x_{2}, t, p_{2}\right) d t+g\left(x_{1}, p_{1} x_{1}+p_{2} x_{2}, p_{2}\right) .
$$

Similarly, if $u \in C^{2}\left(\mathbf{R}_{+}^{4}\right)$ satisfies (5.4), then $u$ has the form

$$
u(x ; p)=\frac{1}{p_{1}} \int_{1}^{p_{2}} \tilde{h}\left(\frac{x_{2}}{t}, p_{1} x_{1}+p_{2} x_{2}, t, p_{1}\right) d t+\tilde{g}\left(x_{2}, p_{1} x_{1}+p_{2} x_{2}, p_{1}\right)
$$

where $\tilde{h} \in C^{2}\left(\mathbf{R}_{+}^{4}\right)$ and $\tilde{g} \in C^{2}\left(\mathbf{R}_{+}^{3}\right)$ enjoy the same properties as $h$ and $g$ in (5.12). Therefore, in case of strong complementarity $(S C), u$ is necessarily written in both the two forms (5.12) and (5.13).

Proof. We first put $w=p_{2} u_{p_{1}}-x_{1} u_{x_{2}}$ so that (5.3) becomes again (5.8). Therefore, there exists a function $h$ satisfying (5.5) such that

$$
w\left(x_{1}, x_{2}, p_{1}, p_{2}\right)=h\left(\frac{x_{1}}{p_{1}}, p_{1} x_{1}+p_{2} x_{2}, p_{1}, p_{2}\right) .
$$

Hence, any solution $u=u(x ; p)$ to the differential inequality (5.3) satisfies

$$
p_{2} u_{p_{1}}\left(x_{1}, x_{2}, p_{1}, p_{2}\right)-x_{1} u_{x_{2}}\left(x_{1}, x_{2}, p_{1}, p_{2}\right)=h\left(\frac{x_{1}}{p_{1}}, p_{1} x_{1}+p_{2} x_{2}, p_{1}, p_{2}\right)
$$

for some function $h$ as in (5.5). We now make another change of variables, namely

$$
\left\{\begin{array} { l } 
{ \xi _ { 1 } = x _ { 1 } } \\
{ \xi _ { 2 } = p _ { 1 } x _ { 1 } + p _ { 2 } x _ { 2 } } \\
{ \xi _ { 3 } = \frac { p _ { 1 } } { p _ { 2 } } } \\
{ \xi _ { 4 } = p _ { 2 } }
\end{array} \quad \left\{\begin{array}{l}
x_{1}=\xi_{1} \\
x_{2}=\frac{\xi_{2}}{\xi_{4}}-\xi_{1} \xi_{3} \\
p_{1}=\xi_{3} \xi_{4} \\
p_{2}=\xi_{4}
\end{array}\right.\right.
$$

and we put $f\left(\xi_{1}, \xi_{2}, \xi_{3}, \xi_{4}\right)=u\left(\xi_{1}, \frac{\xi_{2}}{\xi_{4}}-\xi_{1} \xi_{3}, \xi_{3} \xi_{4}, \xi_{4}\right)$. Again, the change of variables (5.15) is legitime since it defines a one-to-one map between $\left\{(x ; p) \in \mathbf{R}_{+}^{4}\right\}$ 
and $A=\left\{\xi \in \mathbf{R}_{+}^{4} ; \xi_{2}>\xi_{1} \xi_{3} \xi_{4}\right\}$ and the function $f$ is defined in $A$. With this change of variables (5.14) becomes

$$
f_{\xi_{3}}\left(\xi_{1}, \xi_{2}, \xi_{3}, \xi_{4}\right)=h\left(\frac{\xi_{1}}{\xi_{3} \xi_{4}}, \xi_{2}, \xi_{3} \xi_{4}, \xi_{4}\right) \text {. }
$$

By integrating over $\left[\xi_{4}^{-1}, \xi_{3}\right]$ (for any $\xi_{3}>0$ ) we obtain

$$
f\left(\xi_{1}, \xi_{2}, \xi_{3}, \xi_{4}\right)=\int_{1 / \xi_{4}}^{\xi_{3}} h\left(\frac{\xi_{1}}{\xi_{4} t}, \xi_{2}, \xi_{4} t, \xi_{4}\right) d t+g\left(\xi_{1}, \xi_{2}, \xi_{4}\right),
$$

where $g\left(\xi_{1}, \xi_{2}, \xi_{4}\right)=f\left(\xi_{1}, \xi_{2}, \xi_{4}^{-1}, \xi_{4}\right)$. Returning to the $(x ; p)$-variables, the latter becomes

$$
u(x ; p)=\int_{1 / p_{2}}^{p_{1} / p_{2}} h\left(\frac{x_{1}}{p_{2} t}, p_{1} x_{1}+p_{2} x_{2}, p_{2} t, p_{2}\right) d t+g\left(x_{1}, p_{1} x_{1}+p_{2} x_{2}, p_{2}\right)
$$

and (5.12) follows after the change of variable $t \mapsto p_{2} t$ in the integral.

The set of utility functions satisfying condition (SC) or (SS) is quite large. There are utility functions that satisfy both and therefore as the price of a good increases the quantity demanded decreases and the quantity demanded of the other good goes up. An interesting example is the following: consider a function $U \in C^{2}\left(\mathbf{R}_{+}\right)$such that $U^{\prime}>0$ and $U^{\prime \prime}<0$ in $\mathbf{R}_{+}$, take

$$
u(x ; p)=U\left(\frac{x_{1}}{p_{2}}+\frac{x_{2}}{p_{1}}\right) .
$$

For this function $u,(4.5)$ and (4.6) are equivalent to the dual ones. It is easy to show that

$$
\Psi(x, p)=\Phi(x, p)=-\frac{1}{p_{1}} U^{\prime}\left(\frac{x_{1}}{p_{2}}+\frac{x_{2}}{p_{1}}\right)<0,
$$

and therefore $u$ satisfies both (SC) and (SS): if the utility is an increasing function of the sum of the quantities consumed of the different goods divided by their prices then there is good substitution.

\section{Some remarks on the no money illusion property}

Let us first translate the no money illusion property mathematically. Imagine that prices and income are all multiplied by a factor $k>0$. The new budget constraint reads $(x, k p)=(e, k p)$ and is therefore equivalent to $x \in B$, see (2.2). If the maximization of the utility function $(x ; p) \mapsto u(x ; k p)$ under the constraint $x \in B$ gives the same optimum $x^{*}$, then it should have the same level lines (with possibly different levels) as the function $(x ; p) \mapsto u(x ; p)$. Therefore,

$$
\text { for any } k>0 \text { there exists an increasing } f_{k} \in C^{2}(\mathbf{R})
$$

such that $u(x ; k p)=f_{k}(u(x ; p))$. 
This homogeneity condition, which is straightforward in case of a utility function that depends only the quantity of the goods, calls for an interpretation in case of a utility function that depends both on quantities and prices: agents' preferences are affected by relative prices but not by their level. In Section 7 we characterize power utility functions with demand monotonicity, with the previously discussed comparative statics properties, and with price homogeneity of degree zero of the demand.

A large literature has discussed the no money illusion condition when the utility depends upon prices, see $[17,3,33,11,12,8,7,14,15]$. [17, Theorem 2.1] characterizes price dependent utility functions whose corresponding demand function is homogeneous of degree zero in prices and wealth. According to $[17$, p. 503], when $N=2$ these are the functions $u$ which satisfy the differential equation

$$
p_{1} p_{2} u_{x_{1} p_{1}}-p_{1}^{2} u_{x_{2} p_{1}}+p_{2}^{2} u_{x_{1} p_{2}}-p_{1} p_{2} u_{x_{2} p_{2}}=0 .
$$

Namely $u\left(x_{1}, x_{2} ; p_{1}, p_{2}\right)=f\left(x_{1} p_{1}+x_{2} p_{2}\right)+h\left(p_{1} / p_{2}, x_{1}, x_{2}\right)$. Unfortunately, this characterization appears incorrect. First of all, (6.2) admits as a possible solution

$$
u(x ; p)=f\left(\frac{p_{1} x_{1}+p_{2} x_{2}}{p_{1} p_{2}}\right),
$$

a case which is not covered by Kalman's general solution. Second thing, (6.2) does not allow utility functions such as

$$
u(x ; p)=\sqrt{\frac{x_{1}}{p_{1}}}+\sqrt{\frac{x_{2}}{p_{2}}}
$$

which, for $e=(1,1)$, induces the following homogeneous of degree zero optimal consumption bundle

$$
x_{1}^{*}(p)=\frac{p_{1} p_{2}^{2}+p_{2}^{3}}{p_{1}^{3}+p_{1} p_{2}^{2}}, \quad x_{2}^{*}(p)=\frac{p_{2} p_{1}^{2}+p_{1}^{3}}{p_{2}^{3}+p_{2} p_{1}^{2}} .
$$

Therefore, his characterization is not reliable and we stick to $(6.1)^{2}$.

Replying to $[11,12],[14,15,7]$ argue that price dependent utility functions $u$ with no money illusion can be written as a monotonic (price dependent) transformation $\phi^{p}$ (consumer's index of cardinal utility) of a classical quantity dependent utility function $v$ with no effects on the demand:

$$
u(x ; p)=\phi^{p}(v(x))
$$

and that price dependent utility functions of the form

$$
u(x ; p)=f(p) v(x)+g(p)
$$

\footnotetext{
${ }^{2}$ Notice that this problem in Kalman's analysis has not been detected in the papers discussing Kalman's results showing several flaws, see $[7,14,15]$.
} 
satisfy sufficient conditions for the symmetry and negative semidefiniteness of the Slutsky matrix. However, [3] provides an example of a price dependent utility function free of money illusion with a symmetric and negative semidefinite Slutsky matrix that cannot be written as in (6.4). In the next Section we characterize price dependent power utility functions with no money illusion that generate and a demand function monotonic with respect to prices, some of our examples cannot be written as in (6.3) providing a further counterexample to results established in the literature.

\section{Power utility functions}

In this section we provide examples of utility functions of power type such that the demand function satisfies conditions (5.1)-(5.2) or (5.3)-(5.4) as well as the homogeneity assumption (6.1). We take advantage of (4.5) and (4.6) and of Theorems 5.1 and 5.2. We also assume that the derivative of the utility with respect to the quantities of the goods is positive and decreasing, see (2.3).

Conditions (SS) and (SC) are satisfied in case of a power utility function under some parametric restrictions. Note that, with the exception of Example 7.3 , our examples provided below of price dependent utility functions satisfy condition (6.1) under suitable constraints and therefore they are money illusion free (see the demand function of Example 7.2 and 7.3) but cannot be written as an increasing transformation of a classical utility function $\left(u(x ; p)=\phi^{p}(v(x))\right)$, as claimed in [14], or as $u(x ; p)=f(p) v(x)+g(p)$ see [3].

Example 7.1. According to (5.16), a first simple example is given by

$$
u(x ; p)=\left(\frac{x_{1}}{p_{2}}+\frac{x_{2}}{p_{1}}\right)^{d}
$$

for some $d \in(0,1)$. This function satisfies both (SS) and (SC). Moreover, (2.3) and the zero-homogeneity condition (6.1) are satisfied.

\section{Example 7.2. Additive power utility}

Given $\alpha, \beta>0$, consider the function

$$
u(x ; p)=\alpha \frac{x_{1}^{a}}{p_{1}^{c}}+\beta \frac{x_{2}^{b}}{p_{2}^{d}} \quad a, b, c, d \geq 0 .
$$

Notice that $u$ satisfies the homogeneity assumption (6.1) if and only if $c=d$ : prices enter utility in the power form with the same exponent. It is easy to show that

$$
\Psi(x, p)=-\alpha a c \frac{p_{2} x_{1}^{a-1}}{p_{1}^{c+1}}-\beta b \frac{x_{2}^{b-1}}{p_{2}^{d}}+\beta b(b-1) \frac{p_{1} x_{1} x_{2}^{b-2}}{p_{2}^{d+1}} .
$$


The function $\Psi$ is non positive for all $(x, p) \in \mathbf{R}_{+}^{4}$ provided that

$$
0<a, b<1 ; \quad c, d \geq 0 .
$$

Notice that these conditions also ensure that the dual inequality $\tilde{\Psi} \leq 0$ holds true and that (2.3) is satisfied. Hence, these are necessary and sufficient conditions for the utility function in (7.1) to satisfy (5.3)-(5.4) and (2.3). Therefore, strong complementarity requires the classical concavity in goods $(0<a, b<1)$ and that prices enter in the power form with a negative exponent.

We now compute

$$
\Phi(x, p)=\alpha a(1-a-c) \frac{p_{2} x_{1}^{a-1}}{p_{1}^{c+1}}-\beta b \frac{x_{2}^{b-1}}{p_{2}^{d}}
$$

so that $u$ in (7.1) satisfies (5.1)-(5.2) and (2.3) if and only if

$$
0<a, b<1 ; \quad 1-a \leq c ; \quad 1-b \leq d .
$$

Therefore, strong substitution requires the classical condition of concavity in goods and that the exponents of the prices be large enough. These conditions imply the previous ones. Note that if $c=d=0$, i.e., $u$ does not depend on prices, then the function $u$ may satisfy the strong complementarity condition but not the strong substitution condition.

After some computations, one sees that the equations defining the demand functions read

$$
\begin{aligned}
& \left(\frac{\beta b}{\alpha a}\right)^{1 /(1-b)} p_{1}^{(1+c) /(1-b)} p_{2}^{(b+d) /(b-1)}\left[x_{1}^{*}\right]^{(1-a) /(1-b)}+p_{1} x_{1}^{*}=\omega, \\
& \left(\frac{\alpha a}{\beta b}\right)^{1 /(1-a)} p_{2}^{(1+d) /(1-a)} p_{1}^{(a+c) /(a-1)}\left[x_{2}^{*}\right]^{(1-b) /(1-a)}+p_{2} x_{2}^{*}=\omega .
\end{aligned}
$$

Although $x_{1}^{*}$ and $x_{2}^{*}$ may not be easily explicitly determined (except for particular values of the parameters), it is clear from the above equations that

$$
\frac{\partial x_{i}^{*}}{\partial p_{i}}<0, \quad \frac{\partial x_{i}^{*}}{\partial p_{j}}>0 \quad(i, j \in\{1,2\}, i \neq j)
$$

regardless of the values of $c$ and $d$ provided that $0<a, b<1$. Therefore, the utility function (7.1) satisfies both (C) and (S) for any value of $c$ and $d$, also negative ones, provided that it is concave in the quantities of the goods.

\section{Example 7.3. Cobb Douglas utility}

Consider the function

$$
u(x ; p)=\frac{x_{1}^{a} x_{2}^{b}}{p_{1}^{c} p_{2}^{d}} \quad a, b, c, d \geq 0 .
$$


For this function no parameter constraint is needed to ensure (6.1). It is easy to show that

$$
\Psi(x, p)=b(c-a-1) \frac{x_{1}^{a} x_{2}^{b-1}}{p_{1}^{c} p_{2}^{d}}-a c \frac{x_{1}^{a-1} x_{2}^{b}}{p_{1}^{c+1} p_{2}^{d-1}}+b(b-1) \frac{x_{1}^{a+1} x_{2}^{b-2}}{p_{1}^{c-1} p_{2}^{d+1}} .
$$

The utility function $u$ in (7.2) satisfies (2.3) and (SC) (that is, both (5.3)-(5.4)) if and only if

$$
0<a, b<1 ; \quad 0 \leq c \leq a+1 ; \quad 0 \leq d \leq b+1 .
$$

On the other hand,

$$
\Phi(x, p)=a(1-a-c) \frac{x_{1}^{a-1} x_{2}^{b}}{p_{1}^{c+1} p_{2}^{d-1}}+b(a+c-1) \frac{x_{1}^{a} x_{2}^{b-1}}{p_{1}^{c} p_{2}^{d}}
$$

so that $u$ in (7.2) satisfies (2.3) and (SS) (that is, both (5.1)-(5.2)) if and only if

$$
0<a, b<1 ; \quad 0 \leq c, d<1 ; \quad a+c=1 ; \quad b+d=1 .
$$

In this case we have $\Phi=0$. Again, these conditions imply the previous ones.

For the utility function (7.2) one can compute explicitly the demand function and obtain

$$
x_{1}^{*}(p)=\frac{a \omega}{a+b} \frac{1}{p_{1}}, \quad x_{2}^{*}(p)=\frac{b \omega}{a+b} \frac{1}{p_{2}} .
$$

Therefore, (7.2) satisfies both (C) and (S) for any value of $c$ and $d$ provided that $0<a, b<1$.

Example 7.4. Since the differential inequalities (5.1)-(5.2), (5.3)-(5.4) are all linear, it is clear that further examples can be obtained by combining linearly (with positive coefficients) the above examples. Only condition (6.1) should be carefully checked. Of particular interest appears the function

$$
u(x ; p)=\alpha \frac{x_{1}^{a}}{p_{1}^{d}}+\beta \frac{x_{2}^{b}}{p_{2}^{d}}+\gamma \frac{x_{1}^{a} x_{2}^{b}}{p_{1}^{d / 2} p_{2}^{d / 2}}+\delta\left(\frac{x_{1}}{p_{2}}+\frac{x_{2}}{p_{1}}\right)^{d}
$$

for some $\alpha, \beta, \gamma, \delta \geq 0$. This function generalizes both (7.1) and (7.2) allowing us to model agents' behavior involving a strong interaction among quantities and prices. The terms with coefficients $\alpha$ and $\beta$ emphasize the independence between the two goods and their prices: a modification of a price does not directly affect the consumption of the other good. The term with coefficient $\gamma$ measures the dependence between the two goods: the modification of both prices is weighted in the same way. As we have seen in (5.16), the term with coefficient $\delta$ plays in favor of both inequalities (4.5) and (4.6). It is of particular importance since, due to the budget constraint (2.2), it reduces to $\delta\left(\frac{\omega}{p_{1} p_{2}}\right)^{d}$ and therefore represents the relative wealth, namely the wealth normalized by prices. 
For utility functions (7.3) it appears almost impossible to verify directly (C) or (S) in (3.3). On the other hand, conditions (SS) and (SC) are simply verified by taking into account the computations in the previous examples ( $\Phi$ and $\Psi$ depend linearly on $u$ ). More precisely, (5.3)-(5.4)-(2.3)-(6.1) are satisfied if and only if

$$
0<a, b, d<1,
$$

whereas (5.1)-(5.2)-(2.3)-(6.1) are satisfied if and only if

$$
0<a, b, d<1 ; \quad a=b=1-\frac{d}{2} .
$$

Example 7.5. Asymmetric utility

$[10,29]$ consider the case of diamonds and other goods (such as bread). The two goods enter the utility function in completely different manners. This suggests to provide an "asymmetric" example where the two goods have different features for the consumer. For simplicity, given $\alpha, \beta>0$ we consider the function

$$
u(x ; p)=\alpha \frac{x_{1}^{a}}{p_{1}^{c}}+\beta p_{2}^{d} x_{2}^{b} \quad a, b, c, d \geq 0 .
$$

Notice that $u$ cannot satisfy the homogeneity assumption (6.1) whereas (2.3) is ensured provided that $0<a, b<1$. Then,

$$
\Phi(x ; p)=-\beta b p_{2}^{d} x_{2}^{b-1}+\alpha a(1-c-a) \frac{p_{2} x_{1}^{a-1}}{p_{1}^{c+1}}
$$

so that (5.1) is satisfied if and only if $a+c \geq 1$. On the other hand,

$$
\Psi(x ; p)=-\alpha a c p_{2} \frac{x_{1}^{a-1}}{p_{1}^{c+1}}-\beta b p_{2}^{d} x_{2}^{b-1}-\beta b(1-b) p_{1} x_{1} p_{2}^{d-1} x_{2}^{b-2}
$$

so that (5.3) is always satisfied. One can also verify that no choice of the parameters allows to obtain the dual inequalities (5.2) and (5.4) to hold for any $(x ; p) \in \mathbf{R}_{+}^{4}$. The asymmetry consists precisely in not requiring these two inequalities. This means that only an increase of the price of the second good (diamonds) may significantly affect the behavior of the consumer. Finally, notice that (7.4) is increasing with respect to $p_{2}$. This is the so-called Veblen effect.

\section{Example 7.6. Veblen effect}

Theorems 5.1 and 5.2 are obtained under the crucial assumption that the quantity demanded is a decreasing function of price. As we have shown in the introduction, this is not always the case. According to Veblen [38], see also [25, 36], sometimes people judge quality by price, especially for luxury goods. In this case, the monotonicity assumption must be reversed and it should be assumed 
that the demand function is increasing with respect to the price. In this situation, the above arguments remain valid provided that all the inequalities are reversed. In particular, Theorems 5.1 and 5.2 hold if (5.5) is replaced by the following

$$
h \in C^{1}\left(\mathbf{R}_{+}^{4}\right) \quad \text { is such that } h=h(\xi) \text { satisfies } \frac{\partial h}{\partial \xi_{1}} \geq 0 .
$$

Once this is clarified, we note that for the additive utility function in (7.1) or the Cobb Douglas utility in (7.2), the Veblen effect is not obtained regardless of the values of $c$ and $d$. Not only the inequalities opposite to (5.3)-(5.4) and to (5.1)(5.2) cannot be verified but also the quantity demanded of a good is always decreasing with respect to its price.

In most of the above examples the utility function satisfies the following "boundary conditions":

$$
\left\{\begin{array}{l}
\lim _{\left(x_{1}, x_{2}\right) \rightarrow(0,0)} u\left(x_{1}, x_{2} ; p_{1}, p_{2}\right)=0 \text { for all } p_{1}, p_{2}>0 \\
\lim _{\left(p_{1}, p_{2}\right) \rightarrow(\infty, \infty)} u\left(x_{1}, x_{2} ; p_{1}, p_{2}\right)=0 \text { for all } x_{1}, x_{2}>0 .
\end{array}\right.
$$

Although these conditions are not necessary, they appear quite natural since for a zero consumption bundle or for very high prices the utility function tends to vanish.

\section{Multiple goods}

The arguments developed in the previous sections give a hint on how to determine utility functions for $N \geq 2$ goods in case (SC) and (SS). Note that in this setting it is almost impossible to establish conditions for $(\mathbf{S})$ and $(\mathrm{C})$ to hold. We use a projection method which enables us to reduce our study to the case of two goods $(N=2)$.

Our purpose is still to solve (2.1) under the budget constraint (2.2). If we maintain assumptions (2.3), the Lagrange multiplier method implies that the unique optimum $x^{*} \in B$ satisfies (2.4) for some $\lambda>0$. In particular, this yields

$$
p_{i} u_{x_{j}}\left(x^{*} ; p\right)-p_{j} u_{x_{i}}\left(x^{*} ; p\right)=0 \text { for all } i \neq j \text {. }
$$

Let us focus our attention on the case where $i=1$ and $j=2$ so that we are back to (3.1). For any $x \in \mathbf{R}_{+}^{N}$ consider its projections $P x$ onto $\mathbf{R}_{+}^{2}$ and $Q x$ onto $\mathbf{R}_{+}^{N-2}$ so that $P x=\left(x_{1}, x_{2}, 0, \ldots, 0\right)$ and $Q x=\left(0,0, x_{3}, \ldots, x_{N}\right)$. We now argue as if $Q x$ and $Q p$ were constant. Consider the level hypersurface $\Gamma \subset \mathbf{R}_{+}^{N}$ containing the optimum $x^{*}$ :

$$
\Gamma=\left\{x \in \mathbf{R}_{+}^{N} ; u(x ; p)=u\left(x^{*} ; p\right)\right\}
$$

and its projection $P \Gamma$ onto $\mathbf{R}_{+}^{2}$. Let also $P B$ be the projection of $B$ onto $\mathbf{R}_{+}^{2}$; it may be rewritten as

$$
P B=\left\{P x \in \mathbf{R}_{+}^{2} ;(P x, p)=(e-Q x, p)\right\}
$$


Then, $P \Gamma$ is tangent to $P B$ at the point $P x^{*}$. We are so reduced to consider again the case $N=2$. Lemma 4.1 continues to hold and, if we assume (SS), the same arguments of the previous sections lead to (5.1)-(5.2). Similarly, if we assume (SC), we obtain again (5.3)-(5.4).

Therefore, one should view $N \geq 2$ goods as $N(N-1) / 2$ couples of goods and each couple of variables $\left(x_{i}, x_{j}\right)$ should satisfy either (5.1)-(5.2) in case (SS) or (5.3)-(5.4) in case (SC). In turn, Theorem 5.1 (case (SS)) or Theorem 5.2 (case (SC)) hold for any couple of variables. We point out that there may be both couples of type (SS) and of type (SC) at the same time: for instance, $\left(x_{1}, x_{2}\right)$ may satisfy (5.1)-(5.2) whereas $\left(x_{1}, x_{3}\right)$ may satisfy (5.3)-(5.4).

We now give some examples of power utility functions for $N \geq 2$ goods.

Example 8.1. Consider the function

$$
u(x ; p)=\left(\frac{(x, p)}{\prod_{i=1}^{N} p_{i}}\right)^{d}=\frac{(x, p)^{d}}{\prod_{i=1}^{N} p_{i}^{d}}
$$

for some $d \in(0,1)$. We find that for any couple we have $\Phi=0$. Therefore, this function satisfies both (SS) and (SC). Moreover, (2.3) and the homogeneity condition (6.1) are satisfied.

Example 8.2. For given $\alpha_{i} \geq 0$, consider the function

$$
u(x ; p)=\sum_{i=1}^{N} \alpha_{i} \frac{x_{i}^{a_{i}}}{p_{i}^{c_{i}}} \quad a_{i}, c_{i}>0 .
$$

Then, $\Psi(x, p) \leq 0$ for all $(x ; p) \in \mathbf{R}_{+}^{2 N}$ provided that $0<a_{i}<1$ and $c_{i}>0$ which also ensure that (2.3) is satisfied. Hence, these are necessary and sufficient conditions for the utility function in (8.3) to satisfy (SC) and (2.3).

On the other hand, $\Phi(x, p) \leq 0$ for all $(x ; p) \in \mathbf{R}_{+}^{2 N}$ provided that $0<a_{i}<1$ and $1-a_{i} \leq c_{i}$ for all $i=1, \ldots, N$. This is a necessary and sufficient condition for $u$ in (8.3) to satisfy (SS) and (2.3).

Finally, $u$ in (8.3) satisfies the homogeneity assumption (6.1) provided all the $c_{i}$ are equal.

Example 8.3. Take the function

$$
u(x ; p)=\sum_{1 \leq i<j \leq N} \alpha_{i j} \frac{x_{i}^{a_{i}} x_{j}^{a_{j}}}{p_{i}^{c_{i}} p_{j}^{c_{j}}} \quad \alpha_{i j}, a_{i}, c_{i} \geq 0 .
$$

In this case, (6.1) is satisfied if and only if $c_{i}+c_{j}$ is the same for all $i, j$. In turn, this means that all the $c_{i}$ 's are equal. Therefore, (8.4) reduces to

$$
u(x ; p)=\sum_{1 \leq i<j \leq N} \alpha_{i j} \frac{x_{i}^{a_{i}} x_{j}^{a_{j}}}{p_{i}^{c} p_{j}^{c}} \quad \alpha_{i j}, a_{i}, c \geq 0 .
$$


Then, $\Psi \leq 0$ (for any couple $i, j$ ) if and only if $0<a_{i}<1$ and $c \leq a_{i}+1$ for all $i$; this is equivalent to (SC). Moreover, we have $\Phi \leq 0$ for all $i, j$ if and only if also all the $a_{i}$ are equal to some $a \in(0,1)$ which is linked to $c$ by the relation $a+c=1$. Therefore, (8.5) satisfies (SS) if and only if it is of the kind

$$
u(x ; p)=\sum_{1 \leq i<j \leq N} \alpha_{i j} \frac{x_{i}^{1-c} x_{j}^{1-c}}{p_{i}^{c} p_{j}^{c}} \quad \alpha_{i j} \geq 0,0<c<1 .
$$

In this situation, the coefficients $\alpha_{i j}$ become more important: the larger they are, more strict is the link between the two goods $x_{i}$ and $x_{j}$.

In the spirit of (8.4) one can also consider more goods at the same time, with terms of the kind

$$
\prod_{i=1}^{k} \frac{x_{i}^{a_{i}}}{p_{i}^{c_{i}}}
$$

for some $k \leq N$. In this case, the conditions $\Psi \leq 0$ and $\Phi \leq 0$ become more complicated but they are still possible to be verified.

\section{Conclusions}

Utility function dependence on good prices is an old theme. Two points have attracted attention of economists in the '70s: no money illusion (zero homogeneity of the demand in money and price) and price demand effects.

In this paper we have provided sufficient conditions to observe a monotonic demand function and we have shown that under some conditions power utility functions allow us to rule out money illusion, however in these cases price dependent utility functions are not simply an increasing transformation of a classical utility function as claimed in $[7,14,15]$, doing this we go further on the analysis provided by [3]. It is rather difficult to evaluate the effect of a price increase on the quantity demanded of a good, in particular the analysis is difficult in a multiple good setting. In the literature no conditions have been provided. In our analysis we have provided sufficient conditions establishing that a differential condition should be satisfied for all points in the quantity-price space. These conditions allow us to provide a characterization of utility functions with a monotonic demand function or substitution among goods.

\section{REFERENCES}

[1] K. Arrow and F. Hahn, General competitive analysis, North Holland, 1971.

[2] L. S. BAGWEll AND D. BERnheim, Veblen effects in a theory of conspicuous consumption, The American Economic Review 86 (1996), 349-373.

[ 3 ] R. Bamann, D. J. Molina and D. J. Slottje, Budget constraint prices as preference changing parameters of generalized Fechner-Thurstone direct utility functions, The American Economic Review 73 (1983), 411-413. 
[4] E. Barucci, S. Polidoro and V. Vespri, Some results on partial differential equations and Asian options, Math. Models Methods Appl. Sci. 11 (2001), 475-497.

[5] E. Berglas and A. Razin, Preferences, separability, and the Patinkin model: a comment, Journal of Political Economy 82 (1974), 199-201.

[6] E. Casado and J. C. Ferrer, Consumer price sensitivity in the retail industry: latitude of acceptance with heterogeneous demand, European J. Oper. Res. 228 (2013), 418-426.

[7] R. Clower and J. Riley, The foundation of money illusion in a neoclassical micro-monetary model: comment, The American Economic Review 66 (1976), 184-185.

[ 8 ] R. DuSANSKY, Utility function transformations and money illusion: reply and further results, The American Economic Review 70 (1980), 823-825.

[9] R. Dusansky, The demand for money and goods in the theory of consumer choice with money, The American Economic Review 79 (1989), 895-901.

[10] R. Dusansky, Diamonds are a government's best firned: burden free taxes on goods valued for their values: comment, The American Economic Review 79 (1989), 1285-1288.

[11] R. Dusansky and P. Kalman, The foundations of money illusion in a neoclassical micormonetary model, The American Economic Review 64 (1974), 115-122.

[12] R. Dusansky and P. Kalman, The foundations of money illusion in a neoclassical micormonetary model: reply, The American Economic Review 66 (1976), 192-195.

[13] L. Hildebrandt and U. Wagner, Marketing and operations research a literature survey, OR Spektrum 22 (2000), 5-18.

[14] P. HowitT AND D. PATINKIN, Utility function transformations and money illusion: comment, The American Economic Review 70 (1980), 819-822.

[15] P. HowitT AND D. Patinkin, Utility function transformations and money illusion: a further comment, The American Economic Review 70 (1980), 826-828.

[16] N. Ireland, On limiting the market for status signals, Journal of Public Economics 53 (1994), 91-110.

[17] P. J. Kalman, Theory of consumer when prices enter the utility function, Econometrica 36 (1968), 497-510.

[18] P. J. Kalman, Classes of utility functions admitting Tyrni's homogeneous saving function, Review of Economic Studies 36 (1969), 122-124.

[19] P. J. Kalman, R. Dusansky and B. Wickstrom, On the major Slutsky properties when money is the sole medium of exchange, International Economic Review 15 (1974), 718-728.

[20] G. Kalyanaram AND R. Winer, Empirical generalizations from reference price research, Marketing Science 14 (1995), G161-G169.

[21] B. KosZEGI AND M. RABIN, A model of reference-dependent preferences, Quarterly Journal of Economics 121 (2006), 1133-1165.

[22] J. M. LasRy and P. L. Lions, Towards a self-consistent theory of volatility, J. Math. Pures Appl. 86 (2006), 541-551.

[23] J. M. LASRY AND P. L. Lions, Large investor trading impacts on volatility, Ann. Inst. H. Poincaré Anal. Non Lin. 24 (2007), 311-323.

[24] J. M. Lasry and P. L. Lions, Mean field games, Japan. J. Math. 2 (2007), 229-260.

[25] H. Leibstein, Bandwagon, snob, and Veblen effects in the theory of consumers' demand, Quarterly Journal of Economics 64 (1950), 183-207.

[26] B. Mandel, Art as an investment and conspicuous consumption good, American Economic Review 99 (2009), 1653-1663.

[27] A. Mas Colell, M. Whinston and J. Green, Microeconomic theory, Oxford University Press, 1995.

[28] K. Miyagishima, Ranking linear budget sets with different available goods: a note, Math. Social Sci. 63 (2012), 234-238. 
[29] Y. NG, Diamonds are a government's best friend: burden-free taxes on good valued and their values, The American Economic Review 77 (1987), 186-191.

[30] Q. PARIS AND M. CAPUTO, Comparative statics of money-goods specifications of the utility function, Journal of Economics 77 (2002), 53-71.

[31] D. Patinkin, Relative prices, Say's law and the demand for money, Econometrica 16 (1948), 135-154.

[32] D. Patinkin, Money, interest and prices, Harper \& Row, New York, 1965.

[33] R. Pollak, Price dependent preferences, The American Economic Review 67 (1977), 64-75.

[34] D. PUTLER, Incorporating reference price effects into a theory of consumer choice, Marketing Science 11 (1992), 287-309.

[35] P. Samuelson and R. Sato, Unattainability of integrability and definitess conditions in the egneral case of demand for money and goods, The American Economic Review 74 (1984), $588-604$.

[36] T. Scitovsky, Some consequences of the habit of judging quality by price, Review of Economic Studies 12 (1945), 100-105.

[37] I. TyRnI, The effect of price changes on consumer saving, Review of Economic Studies 31 (1964), 149-162.

[38] T. Veblen, The theory of the leisure class: an economic study of institutions, Unwin books, London, 1899.

[39] F. Vermeulen, Collective household models: principles and main results, Journal of Economic Surveys 16 (2002), 533-564.

[40] H. B. YAN, V. N. HUYNH AND Y. NAKAMORI, A group nonadditive multiattribute consumeroriented Kansei evaluation model with an application to traditional crafts, Ann. Oper. Res. 195 (2012), 325-354.

Emilio Barucci

Dipartimento di Matematica

Politecnico di Milano

Piazza Leonardo da Vinci

32-20133 Milano

ITALY

E-mail: emilio.barucci@polimi.it

Filippo Gazzola

Dipartimento di Matematica

Politecnico di Milano

Piazza LeONardo Da VINCI

32-20133 Milano

ITALY

E-mail: filippo.gazzola@polimi.it 